\title{
Método de la correlación en un cristal de $\mathrm{ZnO}$.
}

\author{
Correlation Method in a ZnO Crystal.
}

Oslen Dilayder Jaimes a*
Isabel Cristina Rincón a
Heriberto Peña-Pedraza a

Fecha de Recepción: 16 - oct. - 2017.

Fecha de Aceptación: 17 - abr. - 2018.

\section{Resumen}

La predicción de los modos de vibración de las moléculas o estructuras cristalinas basados en sus propiedades de simetría, nos permiten utilizar un poderoso método para caracterizar nuevos materiales. En el siguiente trabajo, se realizó el análisis modal teórico por medio del método de correlación, y se obtuvo los modos de vibración Raman opticamente activos para un sistema cristalino con estructura de tipo Hexagonal Wurzita. Utilizando la técnica de espectroscopia Raman, se registró el espectro de vibración Raman Stokes para un cristal de $\mathrm{ZnO}$. A partir del análisis teórico y del espectro Raman registrado experimentalmente para el $\mathrm{ZnO}$, se pudo observar y comparar el método teórico (método de correlación) con el experimental (técnica de espectroscopia Raman). Este análisis, sugiere que el método de correlación es preciso en la predicción de los modos de vibración de cualquier red cristalina, sin embargo, su poder se puede extender al usarse conjuntamente con los datos experimentales Raman en la caracterización de la calidad cristalina y estructural de nuevos materiales de interés tecnológico.

Palabras Clave: Modos Normales, Espectroscopia Raman, Wurzita, Correlación.

\begin{abstract}
The prediction of vibrational modes of the crystalline molecules or structures based on their properties of symmetry, allow us to use a powerful method to characterize new materials. In this work, the theoretical modal analysis was performed by the correlation method, and the optically active Raman vibration modes were obtained for a crystalline system with hexagonal Wurzite type structure. Using the Raman spectroscopy technique, the Raman Stokes vibration spectrum was obtained for a $\mathrm{ZnO}$ crystal. From the theoretical analysis and the experimentally recorded Raman spectrum for ZnO, it was possible to observe and compare the theoretical method (correlation method) with the experimental method (Raman spectroscopy technique). This analysis suggests that the correlation method is accurate in predicting the modes of vibration of any crystal lattice, which can be used in conjunction with the Raman experimental data in characterizing the crystalline and structural quality of new materials of technological interest.
\end{abstract}

Keywords: Normal Modes, Raman Spectroscopy, Wurzita, Correlation.

a Grupo de Óptica Moderna, Departamento de Física y Geología, Universidad de Pamplona, Colombia.

* Correo electrónico: oslendjs@unipamplona.edu.co 


\section{INTRODUCCIÓN}

El óxido de Zinc $\mathrm{ZnO}$ es un semiconductor del grupo II-VI, con brecha directa de $3,37 \mathrm{eV}$ a temperatura ambiente y una alta movilidad de electrones. El dopaje natural con oxígeno lo convierte en un semiconductor de tipo n. Posee una gran transparencia, alta movilidad de electrones, brecha ancha y fuerte luminiscencia a temperatura ambiente [1].

Los semiconductores $\mathrm{ZnO}$ han sido ampliamente investigados en muchas complejidades estructurales [2], [3], [5], [6], [7], [8]. Por sus propiedades químicas y físicas sintonizables, potencialmente útiles con aplicaciones en electrónica, optoelectrónica, catálisis, celdas solares, películas delgadas [3],[5],[8]. Se utiliza ampliamente como: electrodo transparente, sensor de gas, ventanas que ahorran energía o que protegen del calor, transistores de película delgada, diodo emisor de luz.

$\mathrm{El} \mathrm{ZnO}$ es muy versatil ya que puede prepararse en volumen, en películas delgadas o formando nanoestructuras (nanoesferas, nanohilos, nanotiras, nanopartículas, etc.) con diferentes métodos de crecimiento [3],[4],[6],[8], [13].

Este material es un candidato para la fabricación de los dispositivos emisores de luz azul o ultravioleta altamente eficientes, debido a la amplia brecha de energía directa y a la gran energía de enlace del exciton. Sin embargo, se ha reconocido que el $\mathrm{ZnO}$ sin dopar muestra una conductividad de tipo $n$, debido a defectos nativos tales como: los intersticiales de $\mathrm{Zn}$, la emisión verde debida a las vacancias de oxígeno, que se introducen por el crecimiento a altas temperaturas y/o la pobre reactividad del oxígeno suministrado en el crecimiento. Normalmente el $\mathrm{ZnO}$ se encuentra en estructuras cúbicas zincblenda y hexagonal wurzita [9]. La estructura hexagonal tiene un grupo puntual de $6 \mathrm{~mm}$ (en notación de Hermann-Mauguin) o $\mathrm{C}_{6 v}$ (notación de Schoenflies), y un grupo espacial $P 6_{3} m c$ o $C_{6 v}^{4}$. Las constantes de red son: $a=3,25 \AA$ y $c=5,2 \AA$; su relación $c / a \sim 1,60$ aproxima al valor ideal para una celda hexagonal 1,633. La ciencia de los materiales necesita investigar la relación entre la estructura y las propiedades de los materiales, el procesamiento y funcionamiento, y proyecta la estructura de un material para conseguir un conjunto predeterminado de propiedades.

Por otro lado, la Espectroscopia Raman, es una herramienta de análisis e investigación importante de los materiales semiconductores. Es una técnica de dispersión de la luz, en la que un fotón incidente interactúa inelásticamente con la red cristalina, y crea o destruye un fotón, el fotón dispersado porta la información acerca de la energía o frecuencia del fonon creado o aniquilado, lo que permite sondear las vibraciónes fundamentales de los cristales, modos normales de vibración o fonones, dando información útil para la identificación química, la caracterización de las estructuras cristalinas, de los enlaces entre los átomos, el medio, los defectos y el estrés de la muestra sólida.

Al combinar la técnica Raman con las características de simetría de la estructura cristalina, la teoría de grupos y el método de correlación, se tiene una herramienta poderosa para el análisis de las propiedades de los materiales cristalinos.

Algunos de los estudios y proyecciones de este material se citaran como sigue:

Nanovarillas de $\mathrm{ZnO}$ dopado con litio, es un buen candidato para la creación de dispositivos nanogeneradores, debido a que dan una respuesta piezoeléctrica mucho más alta que las nanovarillas de $\mathrm{ZnO}$ puras [11].

$\mathrm{El} \mathrm{ZnO}$ es un semiconductor que tiene muchas aplicaciones, como transductores piezoeléctricos, varistores, fósforos luminiscentes, y películas conductoras transparentes [12]. Como emisor de luz el $Z n O$ posee una energía de exciton de $60 \mathrm{meV}$. En electrónica el $\mathrm{ZnO}$ es muy atractivo debido a su alta rigidez dieléctrica y alta velocidad de saturación [2].

Debido a sus propiedades, el $Z n O$ es un material que promete ser usado en diferentes aplicaciones; como en el desarrollo de sensores de gas en forma de películas delgadas, varistores, láseres ultravioleta y visible, y componentes de celdas solares [14]. 


\section{Cristal de ZnO}

El cristal estudiado de $\mathrm{ZnO}$, fue crecido por el método de transporte de vapor, los datos de los espectros Raman no polarizados fueron registrados y facilitados por el Centro de Estudios en Semi-conductores de la Universidad de Los Andes [17], se utilizó la configuración de retrodispersión con un espectrómetro confocal Dilor XY 800, un láser Ar como fuente de excitación de 514,5nm, con una cámara ccd refrigerada con nitrógeno líquido. Las cuasi-partículas conocidas como fonones ópticos presentan una larga vida en el $\mathrm{ZnO}$. El $\mathrm{ZnO}$ adopta una estructura tipo Wurzita con grupo espacial $C_{6 v}$. Se debe tener en cuenta la existencia de impurezas en el crecimiento, ya que estas afectan la simetría del cristal y por lo tanto su energía de vibración.

\section{Método de la correlación}

El método de la correlación fue desarrollado por S. Bhagavantam y T. Venkavantam, en su libro teoría de grupos y su aplicación a problemas físicos [15]. Para aplicar este método se debe conocer la estructura cristalina del material. Para el $\mathrm{ZnO}$ el sistema cristalino es hexagonal, la estructura de la celda unidad es de tipo Wurzita figura 1.

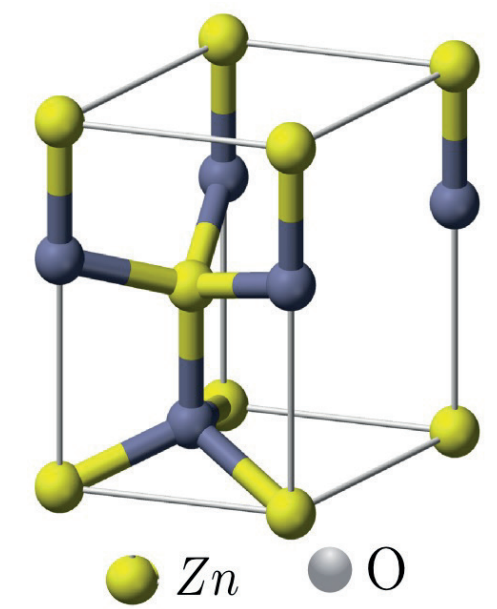

Figura 1. Estructura cristalina tipo Wurzita. Celda unidad del ZnO. Benjah-bmm27

Además, se debe conocer el número de moléculas por celda en el espacio de Bravais, las representaciones irreducibles de una celda unitaria cristalográfica; pueden contener varias veces el número de vibraciónes de la red del cristal. Entonces, la celda de Bravais es utilizada para encontrar las representaciones irreducibles de las vibraciónes en la red. La celda unitaria puede ser idéntica a la celda de Bravais o puede ser mayor en algún múltiplo simple. Para todas la estructuras cristalinas primitivas $(\mathrm{P})$, la celda unitaria y la celda de Bravais son idénticas.

El problema de incluir demasiadas celdas de Bravais en la celda cristalográfica, se soluciona si hacemos la división del número de átomos por celda cristalográfica sobre el núero de puntos en la red (LP) o el número de átomos en cada molécula en la celda cristalográfica.

El número de moléculas por celda unidad: $Z^{n}$ = (número de átomos por celda cristalográfica)/ (número de átomos en cada molécula).

Para la estructura tipo Wurzita 1, el número de átomos por celda cristalográfica es:

$$
N_{Z n}=N_{i}+\frac{N_{c}}{2}+\frac{N_{v}}{8}
$$

donde

$N_{i}$ : Número de átomos en el interior de la celda.

$N_{l}$ : Número de átomos en los lados.

$N_{c}$ : Número de átomos en la cara.

$N_{v}$ : Número de átomos en los vértices.

En la figura $1, N_{i}=1, N_{c}=0$ y $N_{v}=8$

Por lo tanto:

$$
\begin{gathered}
N_{Z n}=2 \\
N_{O}=N_{i}+\frac{N_{l}}{4}+\frac{N_{v}}{8}
\end{gathered}
$$

En la figura $1, N_{i}=1, N_{l}=4 \mathrm{y} N_{v}=0$

Por lo tanto:

$$
N_{O}=2
$$


Entonces tendremos 2 átomos de $Z n$ y 2 de $O$, para un total de 4 átomos en la celda. El número de átomos en la molécula de $\mathrm{ZnO}$ sera 2,1 de $Z n$ y 1 de $O$.

El número de moléculas por celda unidad.

$$
Z_{Z n O}^{n}=\frac{4}{2}=2
$$

\section{Simetría de sitio de cada atomo en la celda de Bravais.}

La posición de cada átomo en la celda unitaria posee su propia simetría, llamada simetría de sitio. Para el $\mathrm{ZnO}$ hay 2 átomos de $\mathrm{Zn}$ y 2 átomos de $\mathrm{O}$ por celda unitaria. El grupo espacial para el $\mathrm{ZnO}$ es el $C_{6 v}^{4}$ o $P 6_{3} m c$ y los posibles sitios de simetría son:

Tabla 1. Simetría de sitio para el grupo espacial P 63mc. Tomado de [16]

\begin{tabular}{cccc} 
Número & Grupo espacial & Grupo puntual & Sitios de simetría posibles \\
\hline \multirow{2}{*}{186} & $P 6_{3} m c$ & $C_{6 v}^{4}$ & $2 C_{3 v}(2) ;$ \\
& & & $C_{s}(6) ; C_{1}(12)$
\end{tabular}

Los valores al lado izquierdo y entre los paréntesis corresponden a:

Tabla 2. Simetría de sitio para el grupo espacial P 63mc. Tomado de [16]

Sitio de simetría Número de átomos posicionados en este en la celda de Bravais sitio de simetría de la celda de Bravais (Número entre paréntesis)
Número de tipos de simetría de sitio (valor al lado izquierdo del grupo puntual)

$\begin{array}{lcc}2 C_{3 v}(2) & 2 & 2 \\ C_{s}(6) & 6 & 1 \\ C_{1}(12) & 12 & 1\end{array}$

El $2 C_{3 v}(2)$ indica que existen 2 tipos diferentes de sitios $C_{3 v}$ en esta celda unidad, cada uno de estos tipos puede posicionar 2 átomos que presentan simetría de sitio $C_{3 v}$. $\mathrm{El} \mathrm{C}_{s}(6)$ indica que existen 6 átomos que ocupan sitios de simetría $C_{s}$.

Para el $\mathrm{ZnO}$ hay 2 átomos de zinc y 2 átomos de oxígeno en la celda unidad. En la tabla 2, la simetría de sitio que puede posicionar 2 átomos es el $C_{3 v}$. Por lo tanto; la simetría del sitio del zinc y del oxígeno es $\mathrm{C}_{3 v}$, el 2 antes de $C_{3 v}$ indica que esta simetría de sitio se repite.

Para seleccionar las simetrías de sitio de los tipos de átomos de una molécula ( $Z n$ y $O)$, se debe tener en cuenta que el número de átomos de un tipo ( $\mathrm{Zn} \mathrm{u} \mathrm{O}$ ) debe ser igual al número de átomos posicionados en el sitio de simetría (valor entre paréntesis) de la tabla 2.

\section{Correlación del grupo del sitio con el grupo factor.}

Ahora se hallarán las especies de simetría para los desplazamientos de los átomos en el sitio. Estos desplazamientos se convertirán en las vibraciónes de la red en el cristal. Las especies de simetría para los sitios de simetría se obtienen por medio de la tabla de correlación (Tabla 6), en nuestro caso sera la correlación entre el grupo puntual $C_{6 v}^{4}$. con la simetría de sitio $C_{3 v}$. Estas tablas muestran la relación entre cada especie del grupo del sitio o el grupo puntual con una especie del grupo factor una simetría de sitio. Entonces, un grupo puntual está correlaciónado con diferentes factores de subgrupos de grupos puntuales, por medio de la simetría de sitio de los átomos en el cristal. Esta correlación permite identificar las especies de vibración de la red en el cristal y además, permite reconocer la actividad Raman e Infrarroja. 


\section{Correlación entre el grupo puntual $C_{6 v}$ y el grupo $C_{3 v^{*}}$}

El grupo $\mathrm{C}_{3 v}$ es un subgrupo de $\mathrm{C}_{6 v}$. Además de este, el grupo puntual también contiene los siguientes subgrupos: $C_{s}, C_{2}, C_{3}, C_{6}$ y $C_{2 v}$. Para mostrar la correlación, se necesitan las tablas de caracteres de los grupos puntuales $C_{6 v}$ y $C_{3 v}$

Tabla 3. Tabla de caracteres para el grupo puntual C6v. Tomada de [16].

\begin{tabular}{l|llllll|l|l}
$C_{6 v}$ & $E$ & $2 C_{6}(\mathrm{z})$ & $2 C_{3}(\mathrm{z})$ & $C_{2}(\mathrm{z})$ & $3 \sigma_{v}$ & $3 \sigma_{d}$ & Lineales y rotaciones & Cuadráticas \\
\hline$A_{1}$ & 1 & 1 & 1 & 1 & 1 & 1 & $\mathrm{z}$ & $x^{2}+y^{2}, z^{2}$ \\
$A_{2}$ & 1 & 1 & 1 & 1 & -1 & -1 & $R_{z}$ & \\
$B_{1}$ & 1 & -1 & 1 & -1 & 1 & -1 & & \\
$B_{2}$ & 1 & -1 & 1 & -1 & -1 & 1 & & \\
$E_{1}$ & 2 & 1 & -1 & -2 & 0 & 0 & $(x, y)\left(R_{x}, R_{y}\right)$ & $(x z, y z)$ \\
$E_{2}$ & 2 & -1 & -1 & 2 & 0 & 0 & & $\left(x^{2}-y^{2}, x y\right)$
\end{tabular}

El grupo puntual $\mathrm{C}_{6 v}$ posee 6 especies de simetría o representaciones irreducibles.

Tabla 4. Tabla de caracteres para el grupo puntual C3v. Tomada de [16].

\begin{tabular}{l|llc|l|l}
$C_{3 v}$ & $E$ & $2 C_{3}(\mathrm{z})$ & $3 \sigma_{v}$ & Lineales y rotaciones & Cuadráticas \\
\hline$A_{1}$ & 1 & 1 & 1 & $\mathrm{z}$ & $x^{2}+y^{2}, z^{2}$ \\
$A_{2}$ & 1 & 1 & -1 & $R_{z}$ & \\
$E$ & 2 & -1 & 0 & $(x, y)\left(R_{x}, R_{y}\right)$ & $\left(x^{2}-y^{2}, x y\right)$
\end{tabular}

El grupo puntual $C_{3 v}$ posee 3 especies de simetría o representaciones irreducibles.

Al comparar las tablas 3 y 4 , se aprecia que las operaciones de simetría del grupo puntual $\mathrm{C}_{3 v}$ están contenidas en el grupo puntual C6v.

Para obtener las especies de $\mathrm{C}_{3 v}$ que están correlaciónadas con las especies de $\mathrm{C}_{6 v}$, se comparan los caracteres de las operaciones de simetría en común de los grupos $\mathrm{C}_{6 v} \mathrm{y} \mathrm{C}_{3 v}$. Estas operaciones de simetría son: E, 2C3(z) y $3 \sigma \mathrm{v}$. Para esto, se toma de la tabla del grupo puntual $\mathrm{C}_{6 v}$ (Tabla 3) solo la parte con las operaciones de simetría en común con el grupo puntual $\mathrm{C}_{3 v}$.
Tabla 5. Caracteres de correlación entre los grupos puntuales C6v y C3v.

\begin{tabular}{|c|c|c|c|c|c|}
\hline $\begin{array}{ll}\text { Especies } & \text { de } \\
\text { simetría } & \text { de } \\
C_{6 v} & \end{array}$ & $E$ & $2 C_{3}(\mathrm{z})$ & $3 \sigma_{v}$ & $\begin{array}{l}\text { Espec } \\
\text { simetr } \\
C_{3 v}\end{array}$ & $\begin{array}{l}\text { es de } \\
\text { ía de }\end{array}$ \\
\hline$A_{1}$ & 1 & 1 & 1 & $A_{1}$ & \\
\hline$A_{2}$ & 1 & 1 & -1 & $A_{2}$ & \\
\hline$B_{1}$ & 1 & 1 & 1 & $A_{1}$ & \\
\hline$B_{2}$ & & 1 & -1 & $A_{2}$ & \\
\hline$E_{1}$ & & -1 & 0 & $E$ & \\
\hline$E_{2}$ & & -1 & 0 & $E$ & \\
\hline & & & $E$ & $2 C_{3}(\mathrm{z})$ & $3 \sigma_{v}$ \\
\hline $\begin{array}{l}\text { Grupo puntus } \\
\text { especie } A_{1}\end{array}$ & alc & $6 v:$ & 1 & 1 & 1 \\
\hline $\begin{array}{l}\text { Grupo puntu } \\
\text { especie } A_{1}\end{array}$ & & & 1 & 1 & 1 \\
\hline
\end{tabular}


Entonces, la correlación es $\mathrm{C}_{3 v}(\mathrm{~A} 1)$ a $\mathrm{C}_{6 v}(\mathrm{~A} 1)$.

\begin{tabular}{l|lll} 
& $E$ & $2 C_{3}(\mathrm{z})$ & $3 \sigma_{v}$ \\
\hline $\begin{array}{l}\text { Grupo puntual } C_{6 v} \text { : espe- } \\
\text { cie } A_{2}\end{array}$ & 1 & 1 & -1 \\
$\begin{array}{l}\text { Grupo puntual } C_{3 v} \text { : espe- } \\
\text { cie } A_{2}\end{array}$ & 1 & 1 & -1
\end{tabular}

La correlación es $C_{3 v}(\mathrm{~A} 2)$ a $C 6 v(\mathrm{~A} 2)$.

\begin{tabular}{l|lll} 
& $E$ & $2 C_{3}(\mathrm{z})$ & $3 \sigma_{v}$ \\
& 1 & 1 & 1 \\
$\begin{array}{l}\text { Grupo puntual } C_{6 v} \text { : espe- } \\
\text { cie } B_{1}\end{array}$ & 1 & & \\
$\begin{array}{l}\text { Grupo puntual } C_{3 v} \text { : espe- } \\
\text { cie } A_{1}\end{array}$ & 1 & 1 & 1
\end{tabular}

La correlación es C3v(A1) a C6v(B1).

Realizando la correlación para las otras

3 especies restantes obtenemos:

Tabla 6. Correlación entre grupos puntuales $\mathrm{C}_{6 v}$ y $\mathrm{C}_{3 v}$.

\begin{tabular}{ll}
$C_{6 v}$ & $C_{3 v}$ \\
\hline$A_{1}$ & $A_{1}$ \\
$A_{2}$ & $A_{2}$ \\
$B_{1}$ & $A_{1}$ \\
$B_{2}$ & $A_{2}$ \\
$E_{1}$ & $E$ \\
$E_{2}$ & $E$
\end{tabular}

Considerando que en los desplazamientos se producen vibraciónes a lo largo de los ejes $(\mathrm{x}, \mathrm{y}, \mathrm{z})$, se pueden identificar de manera fácil las especies de simetría del sitio C3v. Por ejemplo, los desplazamientos de los átomos de zinc $(\mathrm{Zn})$ o de oxígeno $(\mathrm{O})$, paralelos al eje tendrán la misma simetría que la traslación en la dirección z. La traslacion Tz o simplemente $\mathrm{z}$, mostrada en la tabla de caracteres tabla 4 pertenece a la especie de simetría o representación irreducible (IRR) A1. Por lo tanto, los desplazamientos de los átomos a lo largo del eje $\mathrm{z}$, pertenecen a la especie de simetría A1. De la misma manera se puede ver que las traslaciones de los átomos en $\mathrm{x} y \mathrm{y}$ pertenecen a la especie de simetría E tabla 4.

Esta clasificación de las especies de simetría para la vibración por medio de la traslación de los átomos, no es diferente de las descripciones usadas para las vibraciónes moléculares, como: estiramientos, doblamientos y giros. Las vibraciónes moléculares de un cristal son mucho más complejas, pero esa es la importancia de este método, la sencillez con la que clasifica las vibraciónes en la red.

Para encontrar los grados de libertad vibraciónal de las especies de simetría del sitio $\mathrm{C} 3 \mathrm{v}$, que pueden ser tomados para los 2 átomos de zinc $\mathrm{o}$ los 2 átomos oxígeno se usa:

Tabla 7. Grados de libertad de las especies de simetría de sitio C3v. En nuestro caso $n=2$, debido a que son 2 átomos de zinc o también puede ser para 2 átomos de oxígeno.

Especies de Traslación $t^{\gamma}$ Grado de liberSimetría del tad vibracional

\begin{tabular}{llll} 
sitio $C_{3 v}$ & & & $f^{\gamma}=n . t^{\gamma}$ \\
\hline$A_{1}$ & $T_{z}$ & 1 & 2 \\
$A_{2}$ & 0 & 0 & 0 \\
$E$ & $T_{x}, T_{y}$ & 2 & 4
\end{tabular}

La tabla 7 indica que en la red de zinc o de oxígeno, sus vibraciónes son designadas con grados de libertad en las especies $A_{1}$ y E. Ahora vamos a correlaciónar las especies de simetría $\left(A_{1} y E\right)$ del sitio $C_{3 v}$ con las especies del grupo factor $C_{6 v}$, Tabla 6 . 
Tabla 8. Correlación de C3v a C6v.

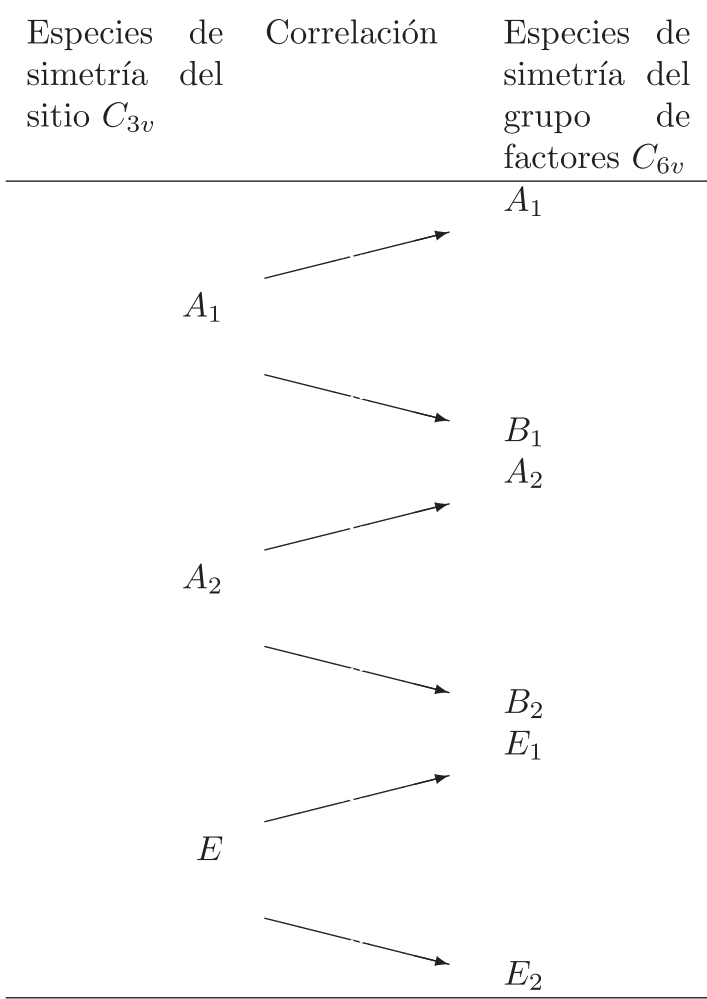

Las especies de simetría de sitio $\mathrm{C} 3 v$ que contienen traslaciones son $A_{1}$ y $E$ (sobre estas traslaciones se pueden producir vibraciónes en la red en el cristal), se selecciona la correlación de estas especies de sitio con las especies de simetría del grupo factor $C_{6 v}$. Por medio de esta selección, es fácil identificar las vibraciónes en la red con especies de simetría del grupo factor, debido a que al considerar las traslaciones de las especies de sitio podemos saber cuáles son las especies de grupo factor que pueden vibrar. Esta correlación permite realizar una conexión entre el movimiento de un átomo en un punto (simetría de sitio) con el movimiento de este átomo en una red en conjunto, debido a que este está enlazado con los demás átomos que forman la celda unitaria (simetría de grupo). Estas especies de simetría de factores son las vibraciónes en la red del cristal. En la tabla 9 se muestran las especies de simetría del grupo factor que están correlaciónadas con las especies de simetría de sitio que contienen traslaciones.

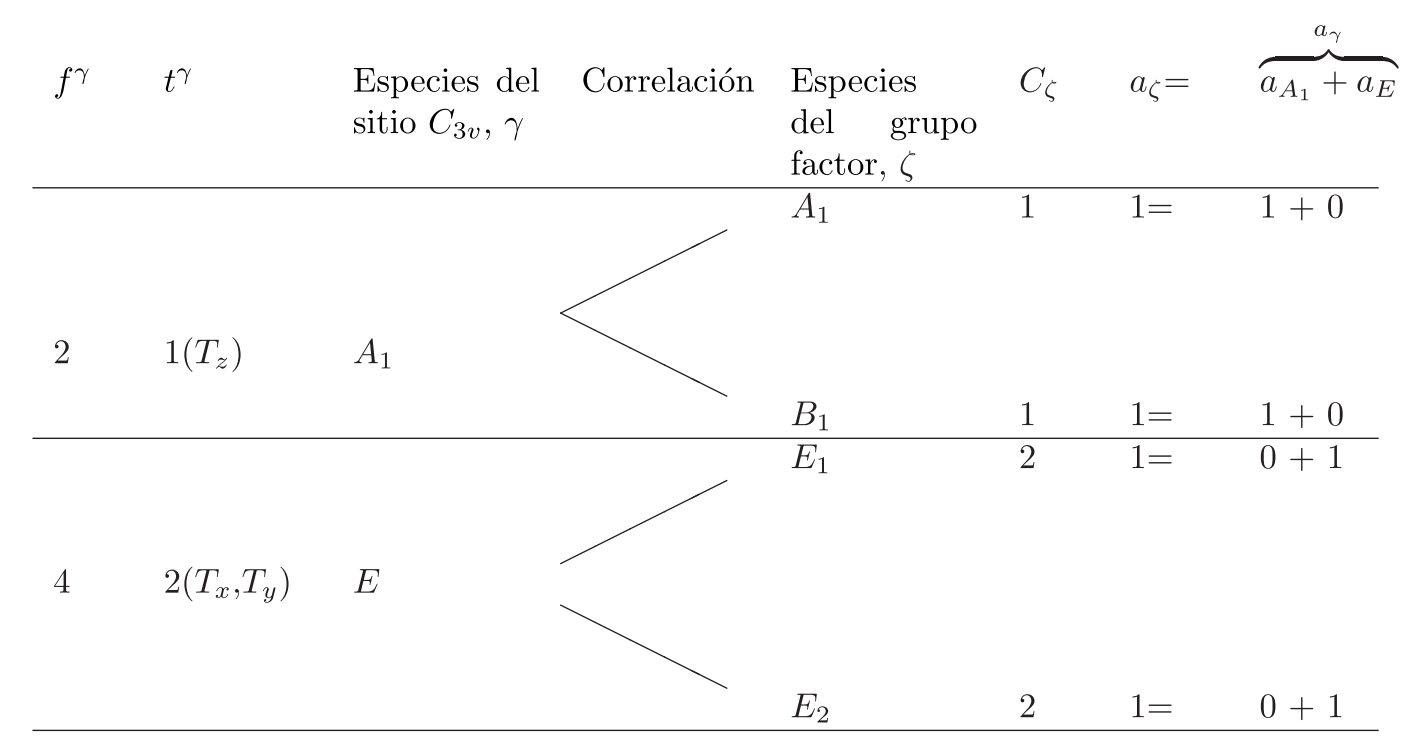

Tabla 9. Valores para encontrar las especies de simetría.

Las representaciones irreducibles de los átomos de Zinc del grupo factor que contiene las vibraciónes en la red son:

$$
\begin{gathered}
\Gamma_{Z n}=1 . A_{1}+1 \cdot B_{1}+1 \cdot E_{1}+1 \cdot E_{2} \\
\Gamma_{Z n}=A_{1}+B_{1}+E_{1}+E_{2}
\end{gathered}
$$

Como tenemos que los átomos de oxígeno también se pueden posicionar en el sitio $\mathrm{C} 3 \mathrm{v}$ y sabemos que este sitio se presenta 2 veces, las representaciones irreducibles para los átomos de oxígeno del grupo factor que contienen las vibraciónes en la red, serán las mismas que para los átomos de zinc, por lo tanto: 


$$
\Gamma_{O}=A_{1}+B_{1}+E_{1}+E_{2}
$$

Se puede encontrar la representacion total pa ra el cristal de $\mathrm{ZnO}$, sumando los 2 conjuntos de representaciones obtenidas:

$$
\begin{aligned}
& \Gamma^{Z n O}=\Gamma_{Z n}+\Gamma_{O} \\
& \Gamma^{Z n O}=2 A_{1}+2 B_{1}+2 E_{1}+2 E_{2}
\end{aligned}
$$

La ecuación de los grados de libertad vibraciónales (Ec. 6) la podemos verificar con el producto de $3 N$, donde $\mathrm{N}$ es el número de átomos en la celda de Bravais o celda unitaria. Para nuestro caso $N=4$ tendremos:

$$
3 * N=3 * 4=12
$$

En la (Ecu 6) tenemos 12 grados de libertad contando el doble degeneramiento de las especies de simetría $E_{1}$ y $\mathrm{E}_{2}$.

En la ecuación de los grados de libertad vibraciónal (Ecu 6), están también incluidos los 3 modos vibraciónales acústicos. Las vibraciónes que se toman en cuenta son las que están en el centro de la zona de Brilloin $(k \cong 0)$, los 3 modos de vibración acústicos en esta zona de Brilloin, tienen una frecuencia aproximada de 0 , las vibraciónes con frecuencia igual a 0 no presentan un interés físico en este estudio, debido a que se estudia la energía absorbida por el cristal para empezar a vibrar. Por lo tanto, estos modos de vibración acústicos pueden ser restados de la (Ecu 6).

$$
\Gamma^{V i b-C r i s t}=\Gamma^{C r i s t a l}-\Gamma^{a c u s t i}
$$

Los modos acústicos se pueden identificar facilmente por medio de la tabla de caracteres para el grupo factor $\mathrm{C}_{6 v}$ Tabla 3, donde las especies de simetría que contengan las 3 traslaciones, le corresponden a los modos acústicos. De esta manera obtenemos que:

$$
\Gamma^{\text {acusti }}=A_{1}+E_{1}
$$

Obteniendo así:

$$
\Gamma^{V i b-C r i s t}=2 A_{1}+2 B_{1}+2 E_{1}+2 E_{2}-\left(A_{1}+E_{1}\right)
$$

$$
\Gamma^{V i b-C r i s t-Z n O}=A_{1}+2 B_{1}+E_{1}+2 E_{2}
$$

Estos modos ópticos se pueden ilustrar en la estructura tipo wurzita figura 2.

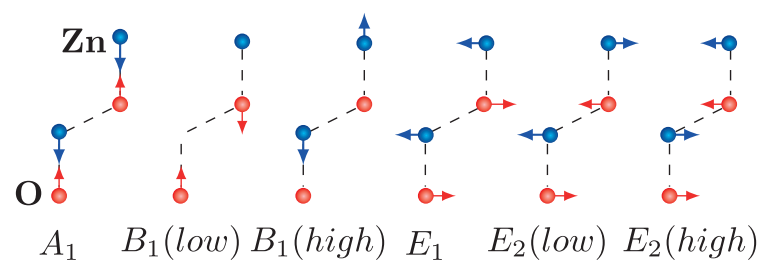

Figura 2. Modos de vibración en la estructura tipo Wurzita.

En la tabla de caracteres para el grupo puntual $\mathrm{C}_{6 v}$ (tabla 3) se puede ver que los modos de vibración B1 son silentes, debido a que esta especie de simetría no presenta actividad IR ni activad Raman. Las especies de simetría $A_{1}$ y $E_{1}$ presen$\tan$ actividad infrarroja. Las especies de simetría $A_{1}, E_{1}$ y $E_{2}$ presentan actividad Raman.

Tabla 10. Actividad IR y actividad Raman en los modos de vibración del $\mathrm{ZnO}$.

\begin{tabular}{|l|l|l|}
\hline$Z n O$ & IR & Raman \\
\hline$A_{1}$ & Activo & Activo \\
\hline$B_{1}$ & No Activo & No Activo \\
\hline$E_{1}$ & Activo & Activo \\
\hline$E_{2}$ & No Activo & Activo \\
\hline
\end{tabular}

De esta manera se pueden obtener los modos normales de vibración del cristal de $\mathrm{ZnO}$ :

$\Gamma^{V i b-C r i s t-Z n O}=A_{1}^{(I R+R)}+2 B_{1}^{(0)}+E_{1}^{(I R+R)}+2 E_{2}^{R}$

\section{RESULTADOS}

Se realizaron los estudios de los modos vibraciónales por espectroscopia Raman del cristal de $\mathrm{ZnO}$, el espectro Raman de la figura 3 fue registrado a temperatura ambiente de $300 \mathrm{~K}$, los datos experimentales del espectro Raman, fueron facili tados para la realización del presente estudio por el Centro de Estudios de Semiconductores, Universidad de los Andes, Mérida-Venezuela y otros colaboradores segun referencia [17]. 


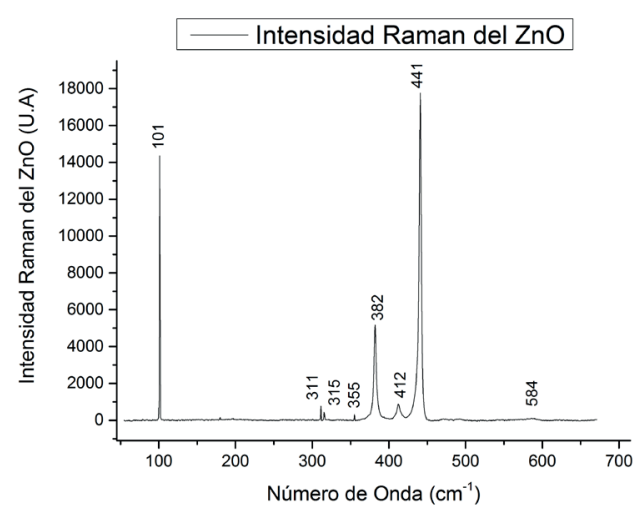

Figura 3. Espectro Raman del $\mathrm{ZnO}$ [17].

El análisis del espectro Raman, se realiza empleando un ajuste tipo Voigt para cada uno de los picos Raman hallados. Determinando así las posiciones energéticas, las intensidades, las áreas y la anchura a media altura (FWHM) utilizando el software comercial (Origin [18]) y la aplicación de funciones tipo Voigt para los picos. El error de este método generalmente es menor del $3 \%$.

Tomando la anchura a media altura (FWHM) y la altura de cada pico tenemos:

Tabla 11. Frecuencia y FWHM de los picos del espectro Raman del cristal de $\mathrm{ZnO}$.

\begin{tabular}{|l|l|l|}
\hline Modos normales & Pico $\left[\mathrm{cm}^{-1}\right]$ & FWHM \\
\hline$E_{2 \text { Low }}$ & 101 & 0.81 \\
\hline$E_{1(\text { TO })}-E_{2 \text { Low }}$ & 311 & 0.42 \\
\hline & 315 & 0.72 \\
\hline$E_{2 \text { High }}-E_{2 \text { Low }}$ & 355 & 1.02 \\
\hline$A_{1(T O)}$ & 382 & 3.54 \\
\hline$E_{1(T O)}$ & 412 & 5.58 \\
\hline$E_{2 H \text { igh }}$ & 441 & 3.39 \\
\hline$A_{1(\text { LO })}$ & Ausente & \\
\hline$E_{1(L O)}$ & 582 & 30.99 \\
\hline
\end{tabular}

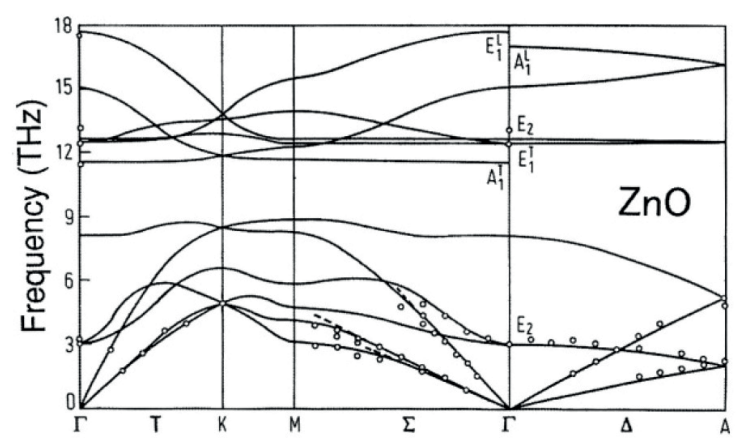

Figura 4. Curvas de dispersión de los fonones del cristal de $\mathrm{ZnO}$. Ref [19].
En el espectro (Fig. 3) se muestran 5 picos que son consistentes con los modos de la curva de dispersión de neutrones (Fig. 4), ( $\mathrm{E}_{2}$ (Low), $\mathrm{A}_{1}(\mathrm{~T}$ $\mathrm{O}), \mathrm{E}_{1}(\mathrm{~T} \mathrm{O}), \mathrm{E}_{2}(\mathrm{High})$ y $\mathrm{E}_{1}(\mathrm{LO})$ ). El modo A1(LO) no aparece en nuestro espectro.

Los picos $311 \mathrm{~cm}^{-1}$ y $355 \mathrm{~cm}^{-1}$ en la Fig 3, se obtienen realizando la combinación lineal de los modos (Ec. 11 y Ec. 12), una razón de esto son los efectos de anarmonicidad o pueden ser provocados por impurezas existentes en el cristal.

$$
E_{1}(T O)-E_{2} L o w=412 \mathrm{~cm}^{-1}-101 \mathrm{~cm}^{-1}=311 \mathrm{~cm}^{-1}
$$

$E_{2} H i g h-E_{2} L o w=441 \mathrm{~cm}^{-1}-101 \mathrm{~cm}^{-1}=340 \mathrm{~cm}^{-1}$

El pico de $315 \mathrm{~cm}^{-1}$ en el espectro de la Fig 3, se atribuye a impurezas o defectos del cristal.

La asignación de los picos de las mediciones Raman es consistente con las curvas de dispersión inelástica de neutrones para el $\mathrm{ZnO}$.

\section{CONCLUSIONES}

Los datos obtenidos experimentalmente de los modos vibraciónales del cristal de $\mathrm{ZnO}$, son muy consistentes con valores reportados por otros autores.

Los fonones encontrados experimentalmente, corresponden a los modos vibraciónales activos Raman identificados teóricamente mediante el uso del método de la correlación y las tablas de caracteres.

Para la estructura tipo Wurzita se dedujeron los modos de vibración con actividad Raman e IR, en el espectro Raman se registraron 8 picos, 5 de estos picos son predichos por el método de la correlación, considerando que los procesos experimentales conllevan una gran cantidad de defectos de la red cristalina, 2 de los picos sobrantes se atribuyen a combinaciones lineales de fonones y el otro a los defectos existentes en el cristal, sin embargo, a pesar de ello, se puede concluir que el cristal de $\mathrm{ZnO}$ estudiado, presenta una buena estructura cristalina. 


\section{REFERENCIAS}

[1] C.J Youn, T.S Jeong, M.S Han, J.H Kim, Optical properties of Znterminated $\mathrm{ZnO}$ bulk. Journal of Crystal Growth, Volume 261, Issue 4, Pages 526-532, 2004.

[2] D. C. Look, Recent Advances in $\mathrm{ZnO}$ Material and Devices, Materials Science and Engineering: B, Vol. 80, No. 1, pp. 383-387. doi:10.1016/S0921-5107(00)00604-8, 2001.

[3] R. S. Wagner and W. C. Ellis, Vapor-Liquid-Solid Mechanism of Single Crystal Growth, Applied Physics Letters, Vol. 4, No. 5, pp. 89-90. doi:10.1063/1.1753975, 1994

[4] Improvement of crystallinity of $\mathrm{ZnO}$ thin film and electrical characteristics of film bulk acoustic wave resonator by using $\mathrm{Pt}$ buffer layer. Vacuum, Volume 74, Issues 3-4, Pages 689-692, 2004.

[5] Y. Polyakov, A.V. Govorkov, N.B. Smirnov, N.V. Pashkova, S.J. Pearton, K. Ip, R.M. Frazier, C.R. Abernathy, D.P. Norton, J.M. Zavada, R.G. Wilson, Y. Li, G. W. Meng, L. D. Zhang and F., Optical and magnetic properties of $\mathrm{ZnO}$ bulk crystals implanted with $\mathrm{Cr}$ and Fe. Materials Science in Semiconductor Processing, Volume 7, Issues 1-2, Pages 77-81, 2004.

[6] G. Tobin, E. McGlynn, M.O. Henry, J.P. Mosnier, J.G. Lunney, D. O’Mahony, E. de Posada, Ultraviolet stimulated emission from bulk and polycrystalline $\mathrm{ZnO}$ thin films with varying grain sizes Physica B: Condensed Matter, Volumes 340-342, Pages 245-249, 2003.

[7] Semiconductor $\mathrm{ZnO}$ Nanowires Arrays and Their Photoluminescence Properties, Applied Physics Letters, Vol. 76, No. 15, pp. 2011-2013. doi:10.1063/1.126238, 2000.

[8] Y. Xia, P. Yang, Y. Sun. Yin, F. Kim and H. Yan, "One Dimensional Nanostructures; Synthesis, Characterization, and Applications," Advanced Materials, Vol. 15, No. 5, pp. 353-389. doi:10.1002/adma.200390087, 2003.

[9] Metal Oxides: Chemistry Applications. Fie rro, J. L. G. CRC Press. p. 182. ISBN 0824723716, 2006.

[10] Ran Cai, Jiagen Wu, Li Sun y Yanjun Liu, 3D graphene/ $\mathrm{ZnO}$ composite with enhanced photocatalytic activity, Materials and Design, 90, pags. 839-844, 2016.

[11] Yu-Tsui Chang, Jui-Yuan Chen, Tzu-Ping Yang, Chun-Wei Huang, Chung-Hua Chiu, Ping-Hung Yeh y Wen-Wei Wu, Excellent pie-zoelectric and electrical properties of lithiumdoped $\mathrm{ZnO}$ nanowires for nanogenerator applications, Nano Energy, 8, pags. 291-296, 2014.

[12] Khalaf Al Abdullah, Sahar Awad, Jean Zaraket and Chafic Salame, Synthesis of $\mathrm{ZnO}$ Nanopowders By Using Sol-Gel and Studying Their Structural y Electrical Properties at Different Temperature, Energy Procedia, volume 119, pages 565 - 570, International Conference on Technologies and Materials for Renewable Energy, Environment and Sustainability, TMREES17, 21-24 April 2017, Beirut Lebanon, issn 1876-6102, doi 10.1016/j.egypro.2017.07.080, 2017.

[13] O.F. Kolomys, V.V. Strelchuk, S.V. Rarata, R. Hayn, A. Savoyant, F. Giovannelli, F. Delorme, V.Tkach, Optical and structural properties of individual Codoped $\mathrm{ZnO}$ microwires, Superlattices and Microstructures, Available online 5 April 2018, ISSN 0749-6036, https://doi.org/10.1016/j.spmi.2018.04.005, 2018

[14] Norton et al.; Chopra et al., 1983; Dayan et al., 1998, Kiriakidis y Katsakaris, 2004.

[15] S. Bhagavantam y T. Venkavantam, Theory of groups and its application to physical problems, 1962.

[16] William G. Fateley y Francis R. Dollish, Infrared and Raman Selection Rules for Molécular and Lattice Vibrations: The Co- 
rrelation Method, WILEY-INTERSGIENCE, pags. 177, 1972.

[17] Marius Millot, Ramon Tena-Zaera, Vicen te Munoz-Sanjose, Jean-Marc Broto y Jesus Gonzalez Anharmonic effects in $\mathrm{ZnO}$ optical phonons probed by Raman spectroscopy,
APPLIED PHYSICS LETTERS, 96, https:// doi.org/10.1063/1.3387843, 2010.

[18] http://www.originlab.com.

[19] A. W. Hewat, Solid State Commun. 8,187, 1970 
